\title{
Patients with primary restless legs syndrome have higher prevalence of autonomic dysfunction and irritable bowel syndrome
}

\author{
Bilgehan Atılgan Acar ${ }^{1}$,MD, Mustafa Atahan Gürkan $\underline{A c a r}^{2}$, MD, Türkan $\underline{A c a r}^{3}$, MD, Ceyhun Varım ${ }^{4}$, MD,
} Aybala Neslihan Alagöz ${ }^{1}$, MD, Enis Bekir Demiryürek ${ }^{3}$, MD, Belma Doğan Güngen ${ }^{3}$, MD, Yeşim Güzey Aras ${ }^{3}$, MD

\begin{abstract}
INTRODUCTION Given the limited data on autonomic dysfunction in patients with primary restless legs syndrome (pRLS), we compared autonomic dysfunction and presence of irritable bowel syndrome (IBS) between patients with pRLS and control patients.

METHODS Consecutive adult drug-naïve patients with pRLS, and age- and gender-matched healthy control patients were enrolled in this study. Diagnoses, based on validated self-reported questionnaires, were made using the following guidelines: Rome III classification system for functional gastrointestinal disorders for IBS; Beck Anxiety Inventory (BAI) and Beck Depression Inventory (BDI) for the presence of anxiety and depression, respectively; Pittsburgh Sleep Quality Index (PSQI) for severity of sleep disturbances; and Scales for Outcomes in Parkinson's disease-Autonomic (SCOPA-AUT) for autonomic dysfunction.

RESULTS There were 88 patients with pRLS ( 18 male, 70 female) and 128 control patients ( 40 men, 88 women). The mean age of the pRLS patients and control patients was $50.3 \pm 9.3$ years and $49.7 \pm 8.2$ years, respectively. Overall, $41(46.6 \%)$ of the patients with pRLS and $16(12.5 \%)$ of the control patients had IBS. Among patients with pRLS, IBS was significantly more common and the total autonomic SCOPA-AUT scores were higher than those found among control patients. Among pRLS patients with IBS, total autonomic SCOPA-AUT, PSQI, BAI and BDI scores were significantly higher than among $\mathrm{pRLS}$ patients without IBS. The presence of IBS did not affect the severity of restless legs syndrome. CONCLUSION The presence of autonomic nervous system impairment in patients with pRLS and the strong link between IBS and pRLS merit further, more extensive investigation.
\end{abstract}

Keywords: autonomic dysfunctions, IBS, RLS, SCOPA-AUT

\section{INTRODUCTION}

Restless legs syndrome (RLS), also known as Willis-Ekbom disease, is a neurological movement disorder characterised by distressing deep sensations in the limbs, particularly the legs, which are associated with an urge to move, often at rest time. ${ }^{(1)}$ RLS is often associated with sleep complaints. ${ }^{(2)}$ Symptoms typically worsen at night or during rest, and resolve with movement. ${ }^{(1)}$ The aetiopathogenesis of RLS is mostly idiopathic, although some secondary aetiologies, such as iron deficiency, pregnancy, uraemia, diabetes mellitus, polyneuropathy, rheumatoid arthritis and spinal disorders, may also be present. ${ }^{(3)}$ The presence of autonomic nervous system impairment in the form of various autonomic symptoms, including increased heart rate ${ }^{(4)}$ and nocturnal blood pressure, ${ }^{(5)}$ erectile dysfunction ${ }^{(6)}$ and effects of dopaminergic treatment, ${ }^{(7)}$ has been described in patients with RLS.

Irritable bowel syndrome (IBS) is a common disorder in primary and secondary care. ${ }^{(8)}$ It is characterised by recurrent abdominal discomfort and pain, and may also be associated with disturbed bowel function. ${ }^{(9)}$ The 2006 Diagnostic Criteria for Functional Gastrointestinal Disorders, third edition, (Rome III) are presently used to define IBS, ${ }^{(10)}$ a chronic functional gastrointestinal disorder resulting from the dysregulation of interactions between the central and enteric nervous systems.

Both RLS and IBS are common diseases with chronically recurring symptoms. RLS affects $9.4 \%-15 \%$ of the general population, ${ }^{(11)}$ while the prevalence of IBS is in the range of $7 \%-21 \%{ }^{(10)}$ Even though the signs and symptoms of these diseases are not malign or fatal by themselves, they do negatively affect quality of life. The pathophysiologies of RLS and IBS are not yet completely clear. Common factors important to the development of RLS and IBS include inflammatory, immune and autonomic functions, and psychosocial status. The complex interactions between the brain and the gastrointestinal tract (brain-gut axis) are believed to be bidirectional and may constitute a common ground for these diseases. ${ }^{(12)}$

Previous studies have investigated the prevalence of RLS among patients with autonomic complaints and IBS. ${ }^{(13-15)} \mathrm{A}$ prospective comparative study by Borji et $\mathrm{al}^{(16)}$ found a significantly higher prevalence of RLS (25\%) among patients with IBS. Furthermore, intensity of discomfort and abdominal pain are more common in patients with IBS and RLS. ${ }^{(16)}$ The prevalence of RLS in patients with other gastrointestinal disorders, such as coeliac disease or Crohn's disease, was found to be $25 \%$ and $30 \%$, 


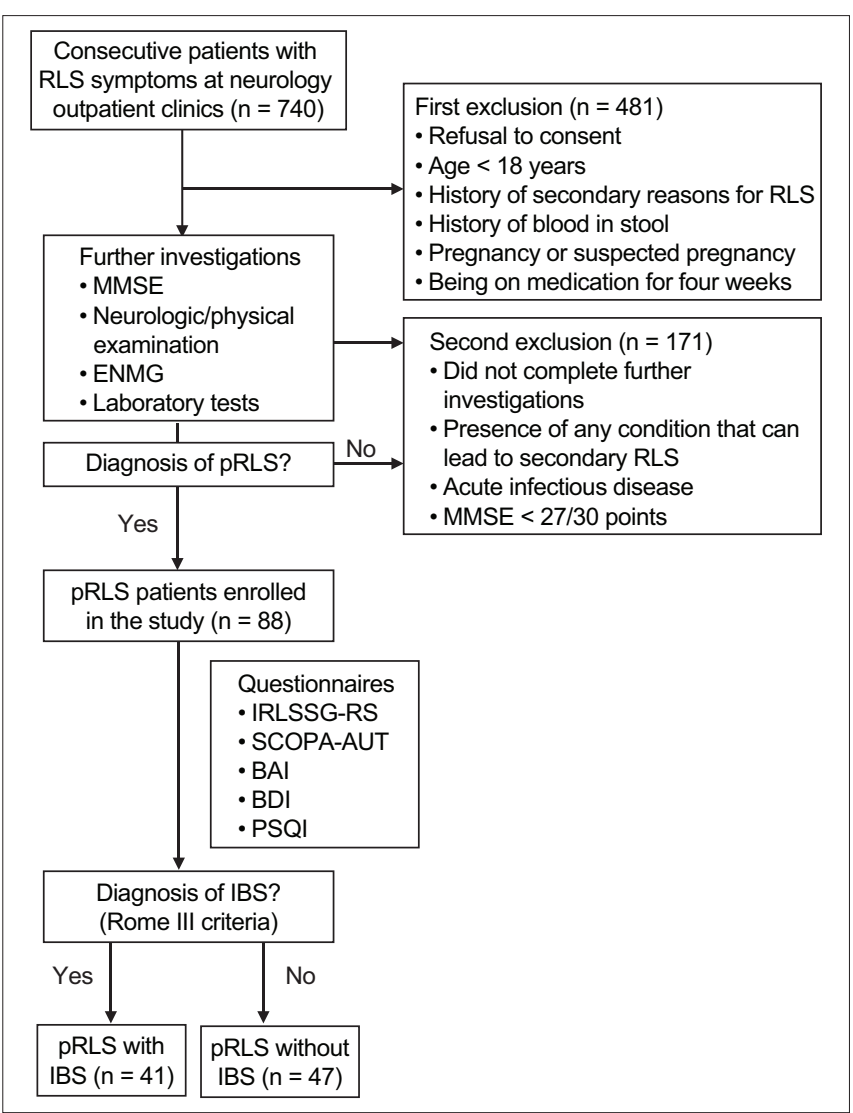

Fig. 1 Flowchart shows the screening process for patients with pRLS. BAI: Beck Anxiety Score; BDI: Beck Depression Score; ENMG: electroneuromyography; IBS: irritable bowel syndrome; IRLSSG-RS: International Restless Legs Syndrome Study Group Rating Scale; MMSE: Mini-Mental State Examination; pRLS: primary RLS; PSQI: Pittsburgh Sleep Quality Index; RLS: restless legs syndrome; SCOPA-AUT: Scales for Outcomes in Parkinson's disease-Autonomic

respectively. ${ }^{(17,18)}$ However, only a few studies with small sample sizes have examined the prevalence of autonomic dysfunction in patients with RLS. ${ }^{(15,19,20)}$ Additionally, Weinstock et al found that the frequency of IBS was $28 \%$ among 32 patients with RLS. ${ }^{(18)}$

In the present study, our main objective was to investigate the autonomic dysfunction of patients with primary RLS (pRLS) when compared to normal control patients using a self-reported questionnaire - Scales for Outcomes in Parkinson's diseaseAutonomic (SCOPA-AUT) - for autonomic dysfunction. ${ }^{(21)}$ A second objective was to compare the occurrence of IBS in patients with idiopathic RLS against matched control patients.

\section{METHODS}

For the test group, 88 consecutive adult patients affected by pRLS were prospectively enrolled from the outpatient clinic at the Department of Neurology, Sakarya University, Sakarya, Turkey, between February 2015 and June 2016. To exclude patients with secondary causes of RLS and ensure the inclusion of only patients with idiopathic RLS, a detailed medical history was taken and neurological examination performed for all patients. Tests included comprehensive movement examination, cognitive examination with the standardised Mini-Mental State Examination (MMSE), electroneuromyography, and routine blood tests for serum urea, serum creatinine, serum iron, total

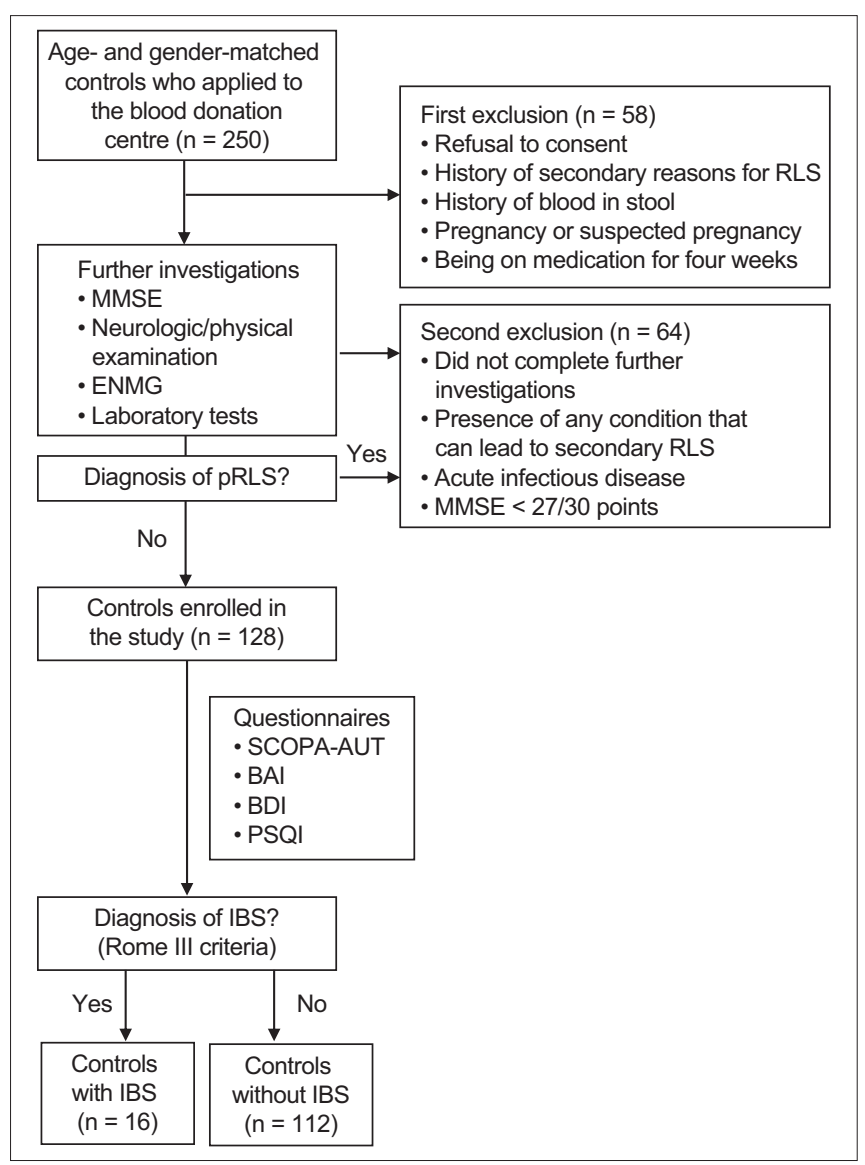

Fig. 2 Flowchart shows the screening process for control subjects. BAl: Beck Anxiety Score; BDI: Beck Depression Score; ENMG: electroneuromyography; IBS: irritable bowel syndrome; MMSE: Mini-Mental State Examination; pRLS: primary restless legs syndrome; PSQI: Pittsburgh Sleep Quality Index; SCOPA-AUT: Scales for Outcomes in Parkinson's disease-Autonomic

iron-binding capacity, serum transferrin and ferritin, erythrocyte sedimentation rate, C-reactive protein, fasting blood glucose and glycated haemoglobin, thyroid function tests, vitamin B12 and folate (Fig. 1). Patients were considered to have pRLS if they met the five essential criteria established by the International Restless Legs Syndrome Study Group (IRLSSG). ${ }^{(22)}$ The IRLSSG Rating Scale includes ten questions related to the severity and frequency of RLS symptoms, with each question on a five-point Likert scale. The total score range for the IRLSSG Rating Scale was 0-40 (mild severity: 0-10, moderate severity: $11-20$, severe: $21-30$, very severe: $31-40){ }^{(23)}$

For the control group, 128 age- and gender-matched patients were selected from among healthy volunteers who had applied to the blood donation centre as blood donors. All control patients underwent neurological and physical examinations, and blood tests similar to patients with pRLS (Fig. 2). None of our control patients showed clinical evidence of RLS and/or systemic disorders.

Patients were diagnosed with IBS according to the Rome III criteria. ${ }^{(24)}$ The Rome III questionnaire was administered, and responses were interpreted by an internist and general surgeon. IBS alone was assessed by the questioning examiner. However, those with abnormal laboratory findings, such as high erythrocyte sedimentation rate, leucocytosis, low level of haemoglobin or a medical history of blood in stool, were excluded. 
Table I. Demographic characteristics, clinical features and autonomic dysfunction status of patients.

\begin{tabular}{|llll|}
\hline \multirow{2}{*}{ Variable } & \multicolumn{2}{c}{ No. $(\%) /$ mean \pm standard deviation } & P-value \\
\cline { 2 - 3 } & pRLS $(\mathbf{n}=\mathbf{8 8})$ & Control $(\mathbf{n = 1 2 8 )}$ & 0.087 \\
\hline Gender & $18(20.5)$ & & \\
\hline Male & $70(79.5)$ & $40(31.3)$ & 0.64 \\
\hline Female & $50.3 \pm 9.3$ & $88(68.8)$ & $<0.0001$ \\
\hline Age $(\mathbf{y r})$ & $41(46.6)$ & $49.7 \pm 8.2$ & 0.97 \\
\hline IBS-positive & $29.0 \pm 5.9$ & $16(12.5)$ & $<0.0001$ \\
\hline Body mass index $\left(\mathbf{k g} / \mathbf{m}^{2}\right)$ & & $29.0 \pm 5.1$ & \\
\hline SCOPA-AUT score & $19.9 \pm 10.5$ & & 0.220 \\
\hline Total & & $14.1 \pm 10.5$ & 0.128 \\
\hline Domain & $3.6 \pm 3.0$ & & $<0.0001$ \\
\hline Gastrointestinal & $5.0 \pm 3.2$ & $3.3 \pm 3.7$ & $<0.0001$ \\
\hline Urinary & $1.6 \pm 1.4$ & $4.7 \pm 3.8$ & $1.0 \pm 1.4$ \\
\hline Cardiovascular & $5.2 \pm 3.2$ & $3.0 \pm 2.4$ & 0.055 \\
\hline Thermoregulatory & $1.0 \pm 0.9$ & $0.8 \pm 0.9$ & 0.005 \\
\hline Pupillomotor & $3.2 \pm 3.5$ & $1.1 \pm 1.3$ & \\
\hline Sexual & & & \\
\hline
\end{tabular}

IBS: irritable bowel syndrome; pRLS: primary restless legs syndrome; SCOPA-AUT: Scales for Outcomes in Parkinson's disease-Autonomic

Exclusion criteria included the following: (a) diagnosis of diabetes mellitus, thyroid disorders, peripheral neuropathy, peripheral vascular diseases, rheumatological diseases, osteoarthritis, Parkinson's disease (PD), chronic kidney disease, anaemia, high ferritin levels or iron deficiency, high erythrocyte sedimentation rate $(>20 \mathrm{~mm} / 2 \mathrm{~h})$, leucocytosis $\left(>10,000 \mathrm{~cm}^{3}\right)$, low haemoglobin levels (female: $<12 \mathrm{~g} / \mathrm{dL}$; male: $<14 \mathrm{~g} / \mathrm{dL}$ ); (b) medical history of blood in stool; (c) pregnancy or suspected pregnancy; (d) being on medication, including dopaminergic, adrenergic or cholinergic agents, antiepileptics, antidepressants, antibiotics or neuroleptics, up to four weeks before enrolment; or (e) a history or clinical finding of dementia or cognitive impairment on the $\operatorname{MMSE}^{(25)}(<27 / 30$ points). These patients were excluded because their accurate completion of SCOPA-AUT could not be guaranteed.

Autonomic dysfunction was evaluated using SCOPA-AUT, which consists of a 23-item questionnaire divided into six domains assessing the following regions: gastrointestinal (seven questions); urinary (six questions); cardiovascular (three questions); thermoregulatory (four questions); pupillomotor (one question); and sexual (two questions for men and two questions for women) dysfunctions. Each autonomic domain was scored in terms of frequency of occurrence, with response options ranging from 0 (never) to 3 (often). All questions included symptoms within the past month, except for syncope (past six months). ${ }^{(21)}$

All participants completed a self-reported validated questionnaire in the Turkish language, including the Beck Anxiety Inventory (BAI), ${ }^{(26)}$ Beck Depression Inventory (BDI) ${ }^{(27)}$ and Pittsburgh Sleep Quality Index (PSQI). ${ }^{(28)}$ The study was approved by the ethical committee at Sakarya University School of Medicine, Sakarya. Informed written consent was obtained from each patient included in the study.

Statistical analysis was done using SPSS Statistics for Windows version 17.0 (SPSS Inc, Chicago, IL, USA). The Kolmogorov-Smirnov test was used as the normality test. Differences between normally distributed continuous data were evaluated using Student's $t$-test. Differences between the two groups of string data were evaluated using chi-square test. Potential factors identified on univariate logistic regression analysis were further evaluated via multivariate logistic regression analysis to determine the independent predictors of IBS. Hosmer-Lemeshow goodness-of-fit test was used to assess the model fit. A percentage Type-I error level was used to infer statistical significance. The PSQI, BAI, BDI and SCOPA-AUT scores were evaluated for predictors of IBS in patients with RLS.

\section{RESULTS}

The mean age of the 88 patients ( 18 male, 70 female) with pRLS and 128 control patients (40 male, 88 female) in our study was $50.3 \pm 9.3$ years and $49.7 \pm 8.2$ years, respectively. There were no statistically significant differences between the two patient groups with respect to demographic variables such as age, gender and mean body mass index. Multivariate logistic regression analysis showed that BAI score (response rate [RR] 0.913, 95\% confidence interval $[\mathrm{Cl}]$ 0.848-0.983; $\mathrm{p}=0.015$ ) and SCOPAAUT-Cardiovascular score (RR 2.665, 95\% Cl 1.437-4.943; $\mathrm{p}=0.002$ ) were found to be predictors for IBS in patients with RLS.

IBS was significantly more common among patients with pRLS than among the control group $(p<0.0001)$. We found that $41(46.6 \%)$ out of 88 patients with pRLS had IBS when compared to $16(12.5 \%)$ out of 128 control patients. The RR for the SCOPAAUT test was $100 \%$. The mean SCOPA-AUT total score among patients with pRLS $(19.9 \pm 10.5)$ was significantly higher than among control patients $(14.1 \pm 10.5 ; p<0.0001)$. There were significant differences between the two groups with respect to the cardiovascular, thermoregulatory and sexual domains. However, there were no statistically significant differences between the gastrointestinal, urinary and pupillomotor functions of the two groups (Table I). 
Table II. Clinical features and autonomic dysfunction status of primary restless legs syndrome (pRLS) patients with and without irritable bowel syndrome (IBS).

\begin{tabular}{|c|c|c|c|}
\hline \multirow[t]{2}{*}{ Variable } & \multicolumn{2}{|c|}{ Mean \pm standard deviation } & \multirow[t]{2}{*}{ p-value } \\
\hline & pRLS patients with IBS $(n=41)$ & pRLS patients without IBS $(n=47)$ & \\
\hline RLS duration (yr) & $11.5 \pm 7.0$ & $10.6 \pm 7.2$ & 0.55 \\
\hline IRLSSG-RS score & $22.1 \pm 10.3$ & $25.4 \pm 6.1$ & 0.58 \\
\hline Body mass index $\left(\mathbf{k g} / \mathrm{m}^{2}\right)$ & $29.4 \pm 4.9$ & $28.9 \pm 5.6$ & 0.53 \\
\hline BAI score & $21.6 \pm 10.6$ & $14.2 \pm 14.0$ & $<0.001$ \\
\hline BDI score & $15.5 \pm 9.8$ & $9.7 \pm 8.4$ & $<0.001$ \\
\hline PSQI score & $9.7 \pm 4.5$ & $6.4 \pm 4.3$ & $<0.001$ \\
\hline \multicolumn{4}{|l|}{ SCOPA-AUT score } \\
\hline Total & $26.2 \pm 6.4$ & $14.4 \pm 10.3$ & $<0.001$ \\
\hline \multicolumn{4}{|l|}{ Domain } \\
\hline Gastrointestinal & $4.9 \pm 3.6$ & $2.9 \pm 3.2$ & $<0.001$ \\
\hline Urinary & $5.8 \pm 4.1$ & $4.5 \pm 3.3$ & 0.035 \\
\hline Cardiovascular & $2.0 \pm 1.6$ & $1.0 \pm 1.4$ & $<0.001$ \\
\hline Thermoregulatory & $5.1 \pm 2.1$ & $3.5 \pm 3.1$ & $<0.001$ \\
\hline Pupillomotor & $1.2 \pm 1.0$ & $0.8 \pm 0.9$ & 0.003 \\
\hline Sexual & $2.9 \pm 3.2$ & $1.6 \pm 2.3$ & 0.008 \\
\hline
\end{tabular}

BAI: Beck Anxiety Score; BDI: Beck Depression Score; IRLSSG-RS: International Restless Legs Syndrome Study Group Rating Scale; PSQI: Pittsburgh Sleep Quality Index; RLS: restless legs syndrome; SCOPA-AUT: Scales for Outcomes in Parkinson's disease-Autonomic

Among patients with pRLS, when assessed in terms of the presence of IBS, there was no statistical difference with respect to the duration $(p=0.55)$ and severity of RLS ( $p=0.58)$, and mean body mass index between pRLS patients with IBS and those without IBS ( $p=0.53$ ). However, pRLS patients with IBS were more anxious and depressive, and suffered worse sleep quality and greater autonomic dysfunction than pRLS patients without IBS (all $p<0.001$ ). Mean SCOPA-AUT total scores and each of its components were statistically higher among pRLS patients with IBS than pRLS patients without IBS. The presence of IBS did not statistically differ between the groups in terms of severity of RLS (Table II).

A number of IBS subcategories were not analysed due to the small sample size of our study. However, complaints of IBS with constipation were more frequent than those of IBS with diarrhoea or the mixed type. None of our patients with pRLS or control patients complained of undetermined IBS.

\section{DISCUSSION}

The aetiology and pathophysiology of RLS and IBS remain incompletely understood. Their aetiologies are most likely multifactorial, involving both biological and psychosocial factors. As earlier stated, the complex interactions between the braingut axis are thought to be bidirectional, constituting a common pathway that is involved in these diseases. ${ }^{(12)}$ The autonomic nervous system is a critical component of this complex central network.

The primary desired outcome of the present study was to evaluate and detect autonomic disturbances among patients with pRLS using the SCOPA-AUT questionnaire. The second outcome was to determine whether IBS was significantly more frequent in patients with pRLS than in control patients. This study posits the presence of increased subjective autonomic complaints in multiple autonomic areas, including cardiovascular, thermoregulatory and sexual areas, among patients with pRLS and the presence of increased frequency of IBS when compared to control patients.

The SCOPA-AUT questionnaire was first used to assess autonomic symptoms in patients with PD. ${ }^{(21)}$ It was reported that increased age, increased disease severity and higher doses of dopaminergic medications were associated with more symptoms of autonomic dysfunction in PD. ${ }^{(29)}$ In previous studies, SCOPA-AUT was independently validated, and researchers have found it to be an acceptable, consistent, valid and precise questionnaire for patients with PD. ${ }^{(30)}$ However, there is limited data regarding autonomic dysfunction in patients with RLS.

Shneyder et al reported that complaints of autonomic system disorders, as measured by SCOPA-AUT, of the gastrointestinal, cardiovascular and pupillomotor domains among 49 patients with RLS and 291 control patients were significantly higher in the former. ${ }^{(19)}$ Using a different methodology, we included only idiopathic patients with RLS through a strict elimination of patients with secondary reasons for RLS. In our study, none of the patients were on medication known to affect autonomic function for at least four weeks prior to inclusion in the study. Another positive aspect of our study was the $100 \%$ RR attained for the SCOPAAUT test. Unlike some retrospective studies, ${ }^{(19)}$ our study found significant differences in the cardiovascular, thermoregulatory and sexual domains of the SCOPA-AUT test between patients with pRLS and control patients, although the gastrointestinal, urinary or pupillomotor functions did not statistically differ between these groups. An interesting point is that while the gastrointestinal domain of SCOPA-AUT did not statistically differ between patients with pRLS and control patients in our study, 
IBS - a very common gastrointestinal disease known to have an autonomic base - was significantly more common in patients with pRLS than in control patients. This allowed us to interpret that control patients had common autonomic-gastrointestinal complaints without a diagnosis of IBS. Additionally, there might be a special relationship between IBS and RLS in addition to their autonomous connection.

The cardiovascular and sexual autonomic complaints of patients with RLS have been previously reported. In a recent study with 23,119 men (944 patients with RLS, 22,175 control patients) who were health professionals, there was a higher frequency of erectile dysfunction among patients with RLS. ${ }^{(6)}$ Another study has reported increased prevalence and incidence of hypertension, cardiovascular diseases and cerebrovascular diseases in patients with RLS. ${ }^{(20)}$ Prior studies have also found positive results in terms of the relation between RLS and cardiovascular autonomic control. ${ }^{(15)}$ Bertisch et al showed that autonomic alterations may relate directly to a higher prevalence of cardiovascular disease in RLS, ${ }^{(15)}$ while Pennestri et al reported that periodic leg movement-related repetitive nocturnal blood pressure fluctuations may contribute to the risk of cardiovascular diseases in patients with RLS. ${ }^{(5)}$ Izzi et al noted that patients with RLS exhibit a tendency towards hypertension, reduced amplitude of both sympathetic systems and parasympathetic responses at the head-up tilt test, and blunted parasympathetic drive to blood pressure changes. ${ }^{(20)}$ These positive correlations between RLS and the sexual and cardiovascular domains of autonomic dysfunction were compatible with our results.

The second objective of our study was to evaluate the impact of IBS on patients with pRLS. We found that pRLS patients with IBS had significantly higher scores for all autonomic subgroups of SCOPA-AUT. Urinary autonomic complaints were also more common in pRLS patients with IBS than those without IBS, although this statistically significant difference was smaller than in the other autonomic subgroups $(p=0.035)$, confirming that pRLS patients with IBS demonstrate severe and nonspecific dysautonomia (Table II). Our results also confirmed that when compared to pRLS patients without IBS, pRLS patients with IBS experienced increased anxiety, depression and sleep disturbance. However, the presence of IBS did not affect RLS severity. Together, these findings reflect the influence of IBS on patients with pRLS from an autonomic and psychological perspective.

The main limitation of our study was its small sample size. Another limitation was the use of questionnaires instead of objective measurements for autonomic functions and the diagnosis of IBS. Additionally, overestimation of results was possible, as the prevalence of autonomic dysfunction and of IBS was calculated from a hospital-based sample. Comparing a possibly overestimated prevalence derived from a highly selected sample with the prevalence among control patients may lead to findings of false statistical significance. Finally, we did not conduct a power analysis prior to the study.

In conclusion, we found that: firstly, patients with pRLS suffer more autonomic complaints in the cardiovascular and sexual domains than similarly assessed control patients in the SCOPA-AUT questionnaire; secondly, IBS, as diagnosed according to the Rome III criteria, was significantly more common in patients with pRLS than control patients, although the gastrointestinal domain of SCOPA-AUT did not statistically differ between patients with pRLS and control patients; and thirdly, the coexistence of pRLS and IBS was associated with high dysautonomia, high depression and anxiety scores, and worse sleep quality, but not RLS severity. The presence of autonomic nervous system impairment in patients with pRLS and the link between IBS and pRLS require further objective and extensive investigations involving larger sample sizes.

\section{REFERENCES}

1. Benes H, Walters AS, Allen RP, Hening WA, Kohnen R. Definition of restless legs syndrome, how to diagnose it, and how to differentiate it from RLS mimics. Mov Disord 2007; 22 Suppl 18:S401-8.

2. Becker PM. The biopsychosocial effects of restless legs syndrome (RLS). Neuropsychiatr Dis Treat 2006; 2:505-12.

3. Allen RP, Walters AS, Montplaisir J, et al. Restless legs syndrome prevalence and impact: REST general population study. Arch Intern Med 2005; 165:1286-92.

4. Gosselin N, Lanfranchi P, Michaud M, et al. Age and gender effects on heart rate activation associated with periodic leg movements in patients with restless legs syndrome. Clin Neurophysiol 2003; 114:2188-95.

5. Pennestri MH, Montplaisir J, Colombo R, Lavigne G, Lanfranchi PA. Nocturnal blood pressure changes in patients with restless legs syndrome. Neurology 2007; 68:1213-8.

6. Gao X, Schwarzschild MA, O'Reilly EJ, Wang H, Ascherio A. Restless legs syndrome and erectile dysfunction. Sleep 2010; 33:75-9.

7. Rocchi C, Albanese M, Placidi F, et al. Chronic dopaminergic treatment in restless legs syndrome: does it affect the autonomic nervous system? Sleep Med 2015; 16:1071-6.

8. Bellini M, Gambaccini D, Stasi C, et al. Irritable bowel syndrome: a disease still searching for pathogenesis, diagnosis and therapy. World J Gastroenterol 2014; 20:8807-20.

9. Tanaka Y, Kanazawa M, Fukudo S, Drossman DA. Biopsychosocial model of irritable bowel syndrome. J Neurogastroenterol Motil 2011; 17:131-9.

10. Chey WD, Kurlander J, Eswaran S. Irritable bowel syndrome: a clinical review. JAMA 2015; 313:949-58.

11. Ohayon MM, O'Hara R, Vitiello MV. Epidemiology of restless legs syndrome: a synthesis of the literature. Sleep Med Rev 2012; 16:283-95.

12. Padhy SK, Sahoo S, Mahajan S, Sinha SK. Irritable bowel syndrome: Is it "irritable brain" or "irritable bowel"? J Neurosci Rural Pract 2015; 6:568-77.

13. Weinstock LB, Fern SE, Duntley SP. Restless legs syndrome in patients with irritable bowel syndrome: response to small intestinal bacterial overgrowth therapy. Dig Dis Sci 2008; 53:1252-6.

14. Basu PP, Shah NJ, Krishnaswamy N, Pacana T. Prevalence of restless legs syndrome in patients with irritable bowel syndrome. World J Gastroenterol 2011; 17:4404-7.

15. Bertisch SM, Muresan C, Schoerning L, Winkelman JW, Taylor JA. Impact of restless legs syndrome on cardiovascular autonomic control. Sleep 2016; 39:565-71.

16. Borji R, Fereshtehnejad SM, Taba Taba Vakili S, Daryani NE, Ajdarkosh H. Association between irritable bowel syndrome and restless legs syndrome: a comparative study with control group. J Neurogastroenterol Motil 2012; 18:426-33

17. Weinstock LB, Bosworth BP, Scherl EJ, et al. Crohn's disease is associated with restless legs syndrome. Inflamm Bowel Dis 2010; 16:275-9.

18. Weinstock LB, Walters AS, Mullin GE, Duntley SP. Celiac disease is associated with restless legs syndrome. Dig Dis Sci 2010; 55:1667-73.

19. Shneyder N, Adler CH, Hentz JG, et al. Autonomic complaints in patients with restless legs syndrome. Sleep Med 2013; 14:1413-6.

20. Izzi F, Placidi F, Romigi A, et al. Is autonomic nervous system involved in restless legs syndrome during wakefulness? Sleep Med 2014; 15:1392-7.

21. Visser M, Marinus J, Stiggelbout AM, Van Hilten JJ. Assessment of autonomic dysfunction in Parkinson's disease: the SCOPA-AUT. Mov Disord 2004; 19:1306-12.

22. Allen RP, Picchietti DL, Garcia-Borreguero D, et al; International Restless Legs Syndrome Study Group. Restless legs syndrome/Willis-Ekbom disease diagnostic criteria: updated International Restless Legs Syndrome Study Group (IRLSSG) consensus criteria--history, rationale, description, and significance. Sleep Med 2014; 15:860-73.

23. Walters AS, LeBrocq C, Dhar A, et al; International Restless Legs Syndrome 
Study Group. Validation of the International Restless Legs Syndrome Study Group rating scale for restless legs syndrome. Sleep Med 2003; 4:121-32.

24. Drossman DA. The functional gastrointestinal disorders and the Rome III process. Gastroenterology 2006; 130:1377-90.

25. Gungen C, Ertan T, Eker E, Yasar R, Engin F. [Reliability and validity of the standardized Mini Mental State Examination in the diagnosis of mild dementia in Turkish population]. Turk Psikiyatri Derg 2002; 13:273-81. Turkish.

26. Ulusoy M, Sahin NH, Erkmen H. Turkish version of the Beck Anxiety Inventory: psychometric properties. J Cogn Psychother 1998; 12:163-72.

27. Hisli N. Beck Depresyon Envanteri'nin üniversite öğrencileri için geçerliği, güvenirliği. Turk Psikol Derg 1989; 7:3-13. Turkish.

28. Agargun MY, Kara H, Anlar O. Pittsburgh Uyku Kalitesi Indeksi'nin Geçerliği ve Güvenirliği. Turk Psikiyatri Derg 1996; 7:107-15. Turkish.

29. Verbaan D, Marinus J, Visser M, et al. Patient-reported autonomic symptoms in Parkinson disease. Neurology 2007; 69:333-41.

30. Rodriguez-Blazquez C, Forjaz MJ, Frades-Payo B, de Pedro-Cuesta J, MartinezMartin P; Longitudinal Parkinson's Disease Patient Study, Estudio Longitudinal de Pacients con Enfermedad da Parkinson Group. Independent validation of the scales for outcomes in Parkinson's disease-autonomic (SCOPA-AUT). Eur J Neurol 2010; 17:194-201. 